\title{
A Study of Infective Endocarditis in Malta
}

\author{
Annalisa Montebello ${ }^{1,}$, , Anette Portelli ${ }^{2}$, Maria Alessandra Zammit ${ }^{1}$, Tonio Piscopo ${ }^{2}$ \\ ${ }^{1}$ Department of Medicine, Mater Dei Hospital, Msida, Malta \\ ${ }^{2}$ Department of Infectious Diseases, Mater Dei Hospital, Msida, Malta
}

Email address:

annalisa.montebello@gov.mt (A. Montebello)

${ }^{*}$ Corresponding author

\section{To cite this article:}

Annalisa Montebello, Anette Portelli, Maria Alessandra Zammit, Tonio Piscopo. A Study of Infective Endocarditis in Malta. Science Journal of Clinical Medicine. Vol. 6, No. 6, 2017, pp. 98-104. doi: 10.11648/j.sjcm.20170606.11

Received: August 15, 2016; Accepted: October 7, 2016; Published: October 30, 2017

\begin{abstract}
Infective endocarditis (IE) is a life threatening infection, especially if diagnosis and subsequent treatment are delayed. The aim of this retrospective study was to assess the clinical features and management of infective endocarditis in Malta. Patients admitted to Mater Dei Hospital (MDH) between January 2008 and December 2015 with a diagnosis of IE according to the modified Duke's criteria were selected from electronic case summaries. Demographic data and details on clinical features, risk factors, investigations, management and complications were obtained through the patients' clinical notes and online laboratory results. There were 65 patients with a diagnosis of IE with a $12.3 \%$ (8) mortality rate. Our cohort included 57 patients as the eight deceased patients were not audited due to unavailable case notes. Native valve endocarditis was seen in $80.7 \%$ (46) of patients whilst 19.3\% (11) had prosthetic valve endocarditis. Left sided vegetations were the most common at $68.4 \%$ (39). The majority of patients were culture positive at $87.7 \%$ (50). Staphylococci were the commonest organisms cultured at $36.8 \%$ (21) of which $57.1 \%$ (12) were Staphylococcus aureus with $19.0 \%$ (4) being Methicillin resistant staphylococcal aureus. Complication rates were highest in the native valve cohort at $67.4 \%$ (31) versus $36.4 \%$ (4) patients in the prosthetic valve endocarditis group. IE is not uncommon and can result in severe complications can arise with long-term sequelae. Preventive efforts should be of utmost importance both in the community and in hospitals.
\end{abstract}

Keywords: Infective Endocarditis, Clinical Features, Investigations, Complications

\section{Introduction}

Infective Endocarditis (IE) is a life threatening infection of the heart endocardium, heart valves or septal defects. It can result in multiple complications including congestive cardiac failure, valvular insufficiency, myocardial abscesses and even death [1].

IE can present with various symptoms and signs. Chills and rigors, low appetite and weight loss are the commonest symptoms. Fever is the commonest symptom as it is present in $90 \%$ of patients followed by heart murmurs found in $85 \%$ of patients [1]. The classical signs of infective endocarditis are found in at least $50 \%$ of patients. These include petecchiae, splinter haemorrhages, osler's nodes, janeway lesions or roth spots. Other signs of IE include splenomegaly, stiff neck, delirium, paralysis, hemiparesis, aphasia, conjunctival haemorrhage, pallor, gallops, rales, cardiac arrhythmias, pericardial rub and pleural friction rub [2].
IE is divided into fourmain types: native valve endocarditis, prosthetic valve endocarditis andintravenous drug abuse (IVDA) related endocarditis and in relation to implantable electronic device infections [2]. Infective Endocarditis is diagnosed using the modified Duke's Criteria. These criteria diagnose IE with $80 \%$ overall sensitivity [3].

\section{Aim}

To assess the number, clinical presentation, risk factors, investigations and management of all patients diagnosed with infective endocarditis in Malta between 2008 and 2015.

\section{Methods}

All patients that were admitted with a diagnosis of infective endocarditis to Mater Dei Hospital between 2008 and 2015 were audited in this retrospective study. Data 
protection approval was sought and case notes were reviewed. Data collected included patient's age, gender, length of stay, signs and symptoms on presentation, risk factors, investigations done, antibiotics used and complications identified.

\section{Results}

65 patients were admitted with a diagnosis of IE with a $12.3 \%$ (8) mortality rate. The deceased patients were not audited as case notes were unavailable.

Our cohort included 57 patients. The majority of patients were males at $63.1 \%$ (36) and $36.8 \%$ (21) were females. (Figure 1) The mean age of the cohort was 58.7 years. The majority of cases occurred in the 50-60 year age group at $24.6 \%$ (14). Native valve endocarditis was more common at $80.7 \%$ (46) whilst $19.3 \%$ (11) had prosthetic valve endocarditis. (Figure 3) The most common site for vegetations was left sided at $68.4 \%$ (39), $14.0 \%$ (8) were right sided, $8.8 \%$ (5) had no vegetations on echocardiogram, $3.5 \%$ (2) had both right and left sided vegetations on echocardiogram $1.8 \%$ (1) implantable cardiac electronic device endocarditis and 3.5\%(2) had vegetation attached to a central venous catheter. (Figure 4)

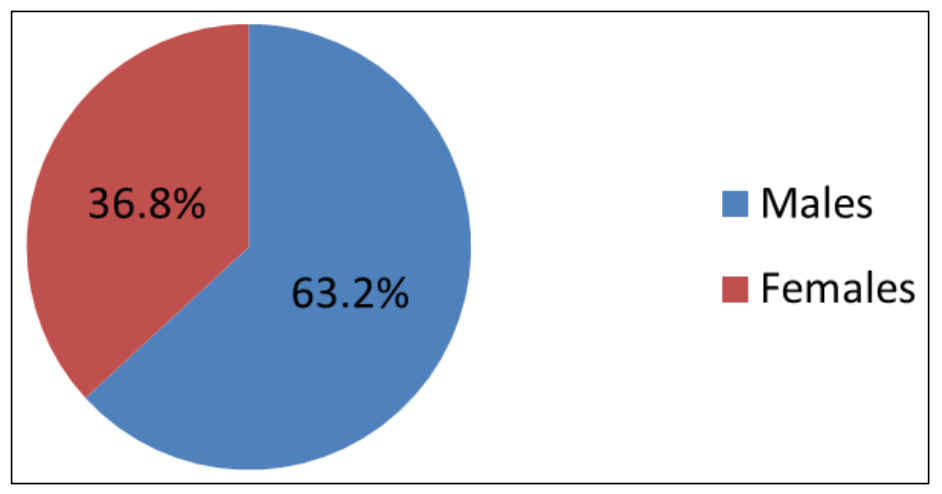

Figure 1. Cohort Gender.

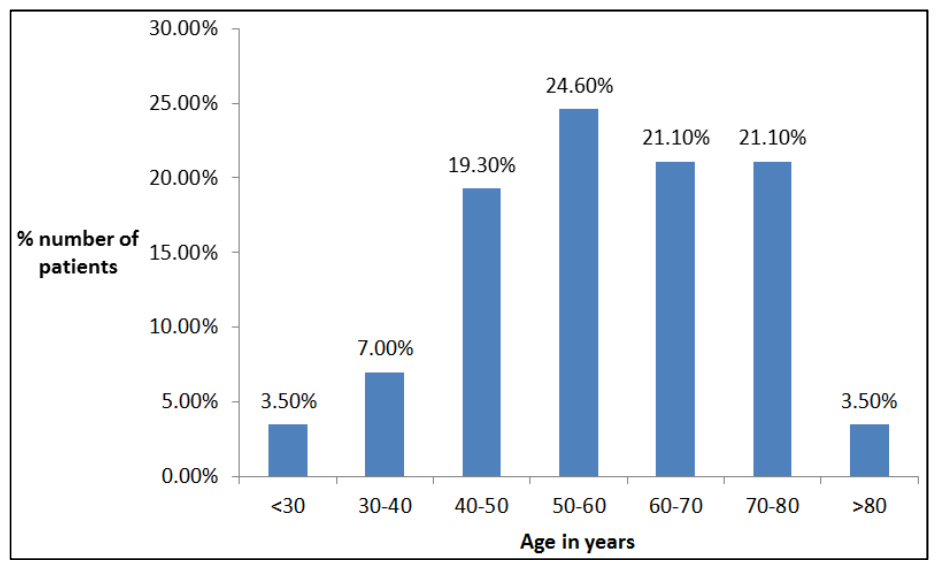

Figure 2. Cohort age distribution.

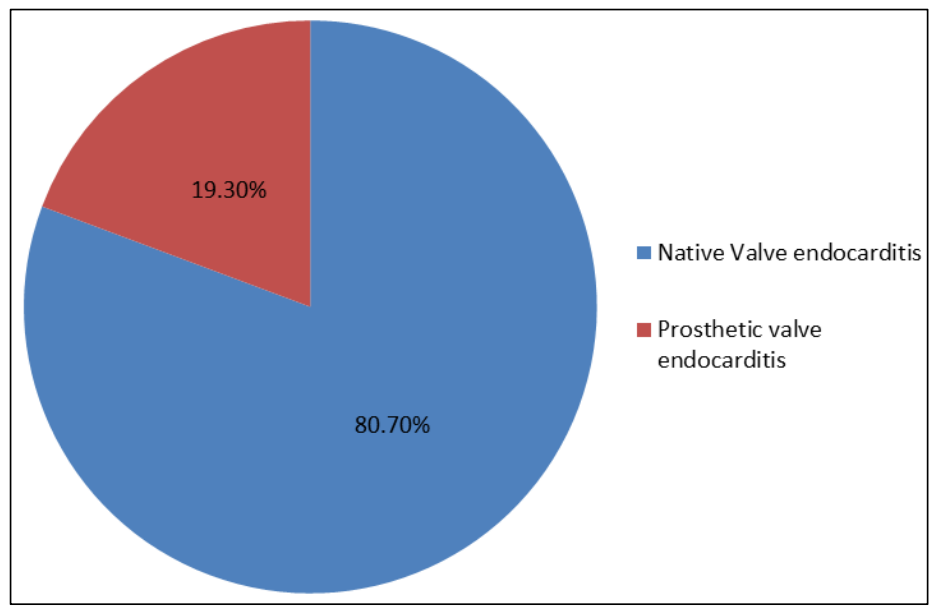

Figure 3. Native vs Prosthetic valve endocarditis. 


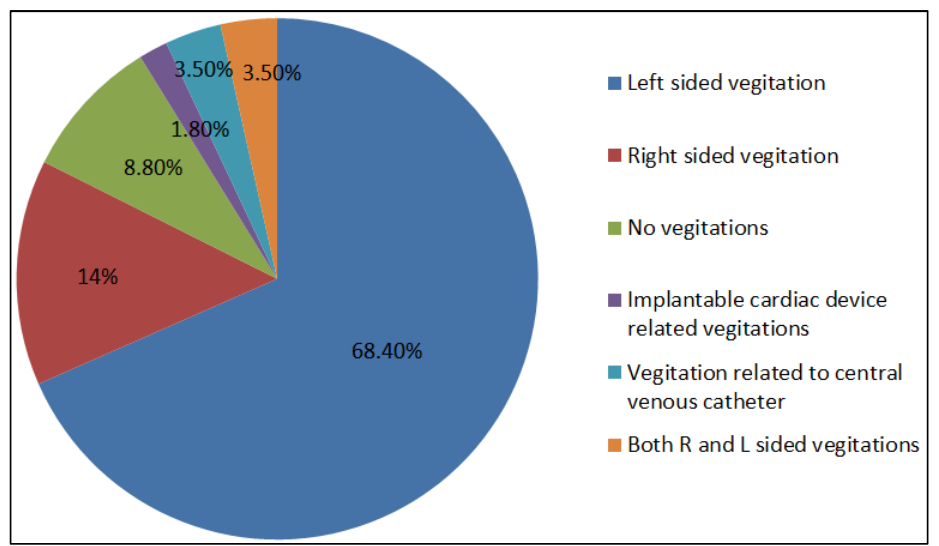

Figure 4. Echocardiogram results.

Risk factors for IE included prosthetic valves at $19.3 \%$ (11), intravenous drug abuse at $12.3 \%$ (7), recent surgical procedures at $5.2 \%$ (3) including colorectal surgery, lower limb amputation secondary to osteomyelitis and tracheostomy insertion, indwelling vascular devices including permcaths and port-a-caths at $8.8 \%$ (5), implantation of cardiac electronic devices at $5.3 \%$ (3), previous cardiac intervention including patent foramen ovale repair at $1.8 \%$ (1), dental procedures at $10.5 \%$ (6) congenital heart defects including VSD at $5.3 \%$ (3) rheumatic heart disease at $3.5 \%$ (2). There was $1.8 \%$ (1) patient that had colon cancer diagnosed during admission and suffered infective endocarditis with Streptococcus gallolyticus. 31.2\% (18) had no risk factors. (Figure 5)

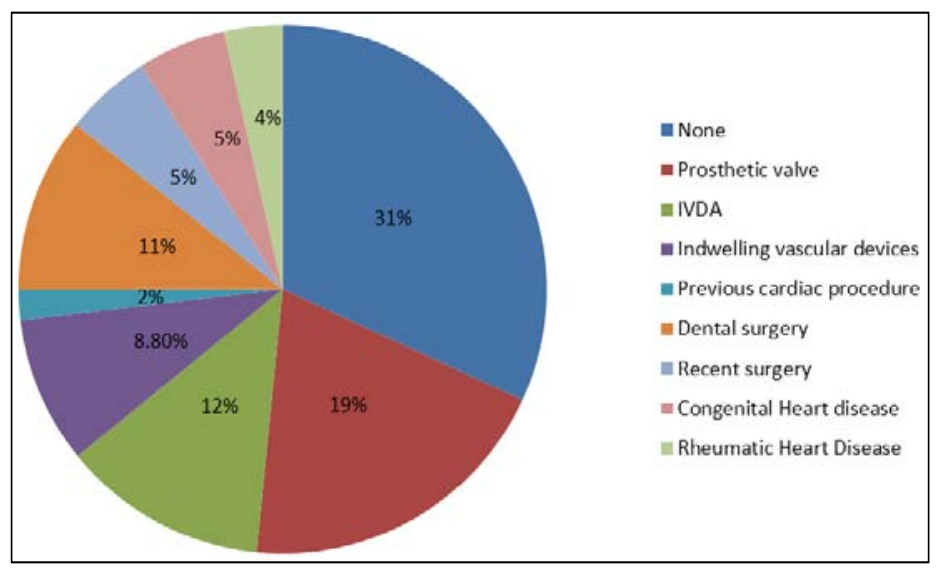

Figure 5. Risk factors for IE.

There were $87.7 \%$ (50) of patients that had culture positive endocarditis. Staphylococci were the commonest organisms cultured at $42.0 \%$ (21) of which $57.1 \%$ (12) were Staphylococcus aureus with $19.0 \%$ (4) being Methicillin resistant staphylococcal aureus. The rest of the staphylococci included Staphylococcus epidermidis, Staphlococcus lugdenensis, Staphylococcus capitis and coagulase negative staphylococci. Other organisms included viridans streptococci at $31.5 \%$ (18) enterococci $8.0 \%$ (4) other gram positive organisms at $4 \%$ (2) including Streptococcus agalactiae, and capsulated streptococcus pneumoniae, other gram negative organisms at $10.0 \%$ (5) including Escherichia coli, Cardiobacterium hominis and Aeromonas hydrophila. $12.3 \%$ (7) blood cultures resulted in no growth. (Figure 6)

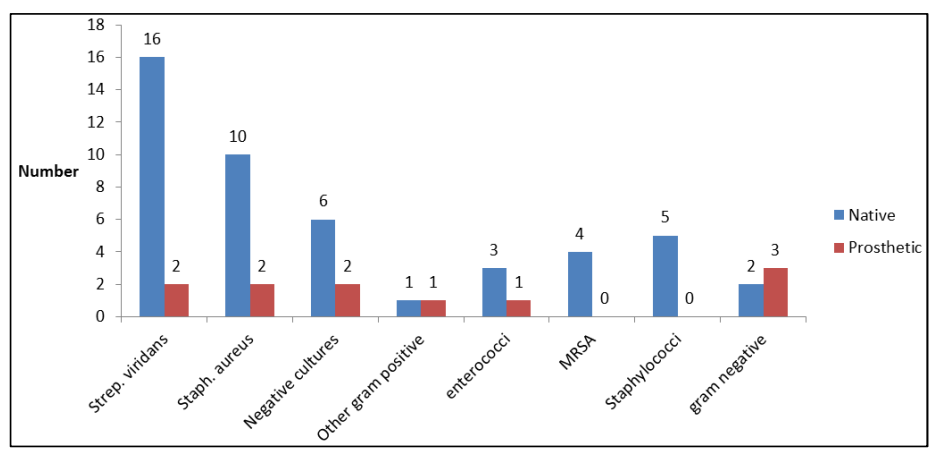

Figure 6. Organisms cultured. 
There were $67.4 \%$ (31) of patients in the native valve endocarditis cohort that suffered complications; $28.3 \%$ (13) of patients suffered septic emboli including brain, lung and spleen emboli, 15.2\% (7) needed valve replacement surgery, $10.9 \%$ (5) needed removal of indwelling venous catheters, $8.7 \%$ (4) required removal of cardiac devices, $4.3 \%$ (2) had pericardial effusion requiring drainage, $2.0 \%$ (1) had discitis and $2.0 \%$ (1) suffered septic shock. $4.0 \%$ (2) had both septic emboli and valve repair. In the prosthetic valve endocarditis cohort $27.3 \%$ (3) had septic emboli, 9.1\% (1) needed valve replacement

Four patients in our cohort between January 2008 and December 2015 had a second episode of IE whereas one patient had three episodes. In all cases the organisms cultured were different in the recurrent episodes.

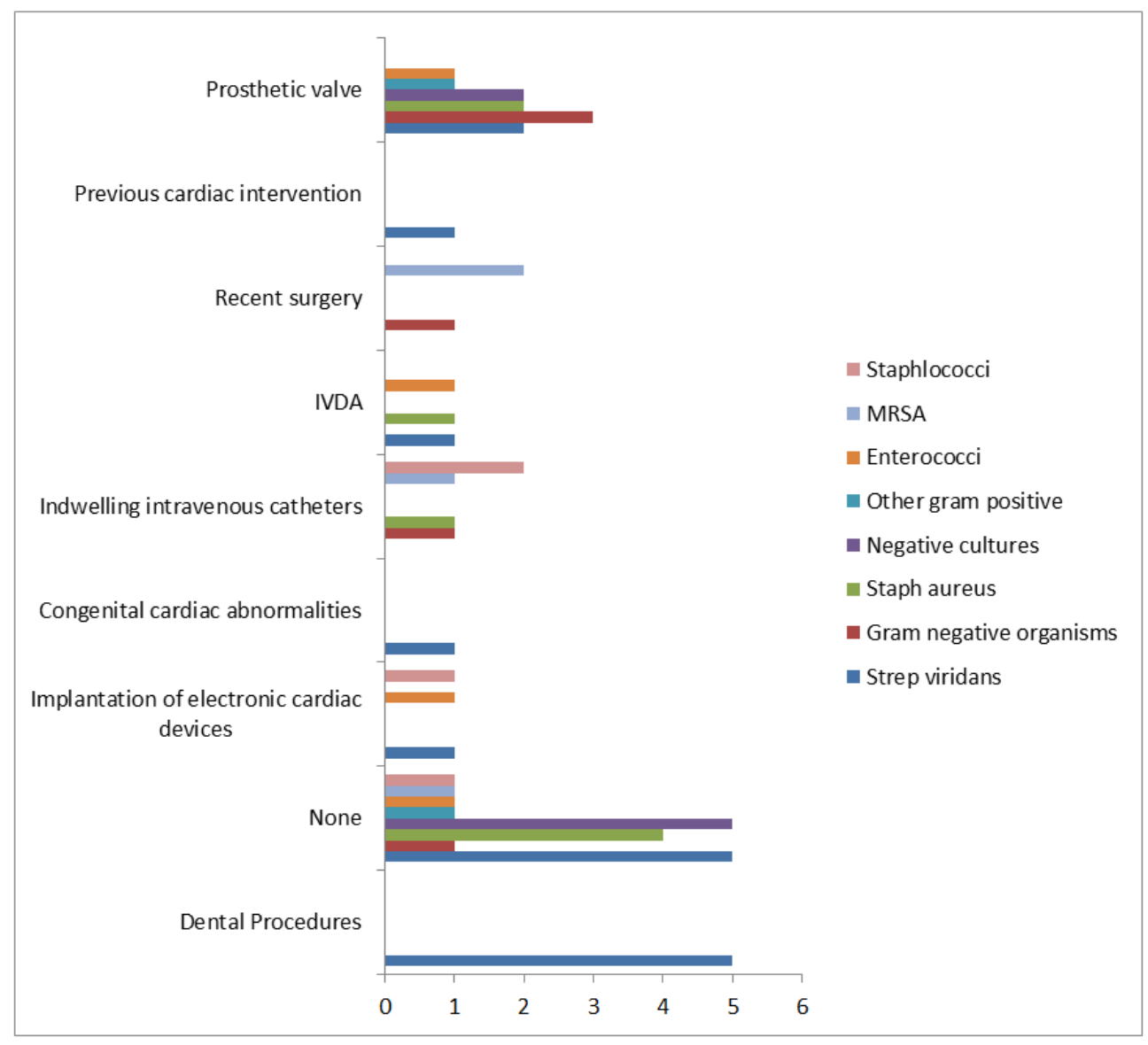

Figure 7. Correlation of microbiology with risk factors.

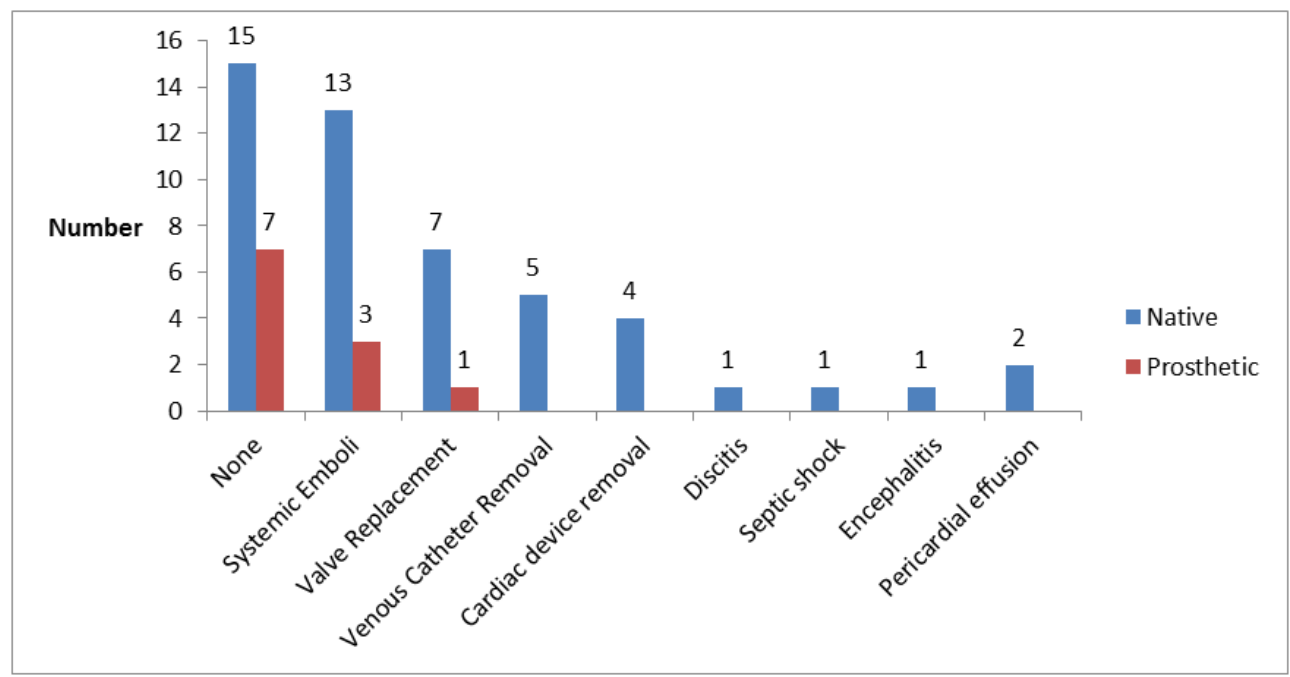

Figure 8. Complications due to IE. 


\section{Discussion}

There has been an increase in the number of IE cases in Malta throughout the years with just 5 cases being diagnosed in 2008 compared to 18 cases in 2015 . The majority of patients were 50 - 60 year old males. The commonest type of IE diagnosed was native valve left sided endocarditis. (Figure 9)

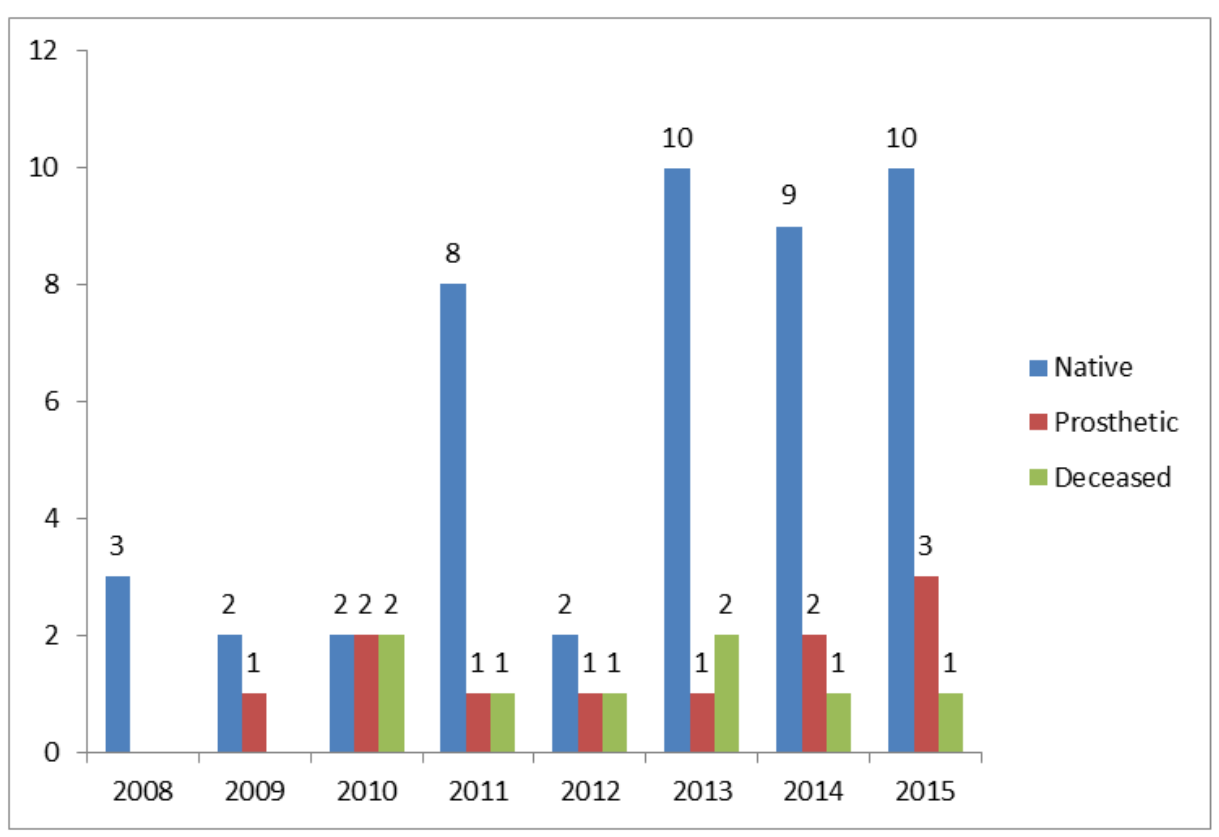

Figure 9. Incidence of IE by year.

Clinical features in infective endocarditis can vary widely and are non-specific. The commonest symptoms include fever, chills and rigors, anorexia and weight loss. Others include malaise, headache, myalgias, arthralgias, night sweats, abdominal pain, dyspnoea, cough and pleuritic pain. The most common symptom in this study was fever at $75.4 \%$ (43), followed by chills and rigors at $40.4 \%$ (23) and weight loss at $12.3 \%$ (7). (Figure 10)

The commonest sign of IE is the presence of cardiac murmurs occurring in $85 \%$ of patients. Petecchiae were seen in $20-40 \%$ of patients. Other signs include splenomegaly, splinter haemorrhages. Uncommon signs include janeway lesions, osler nodes and Roth spots. The latter are found in only $2 \%$ of patients. [1]

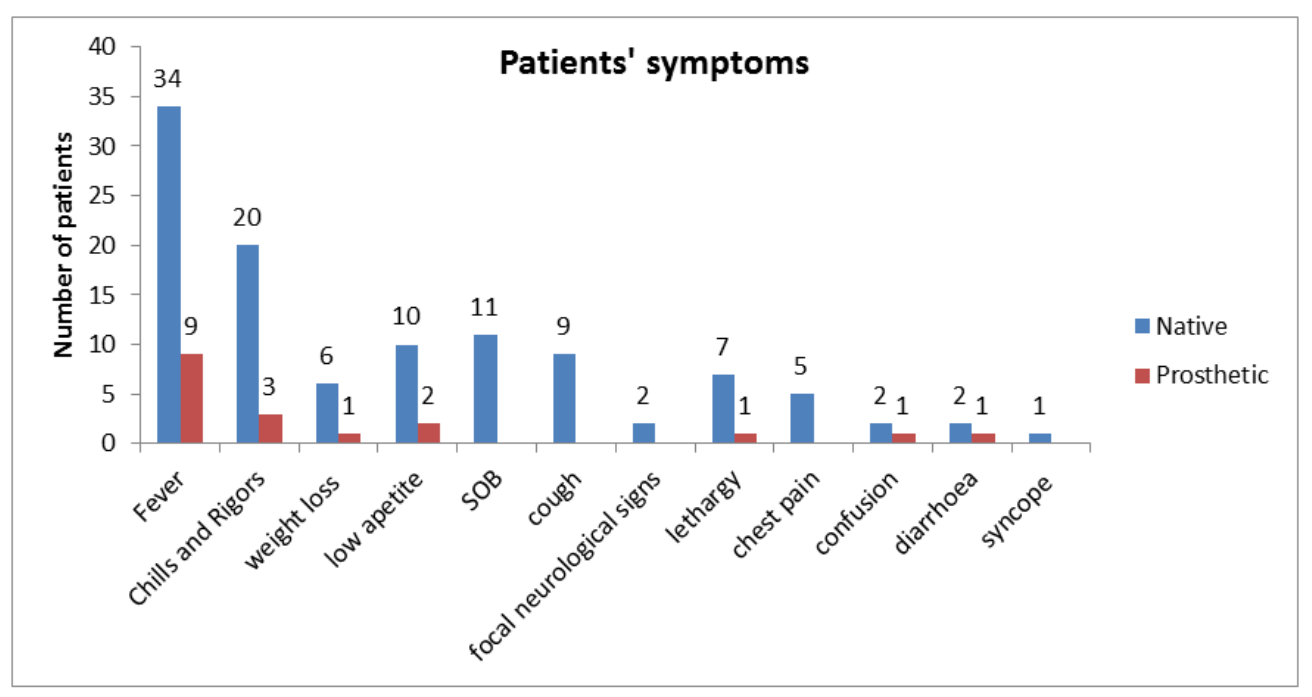

Figure 10. Patients' symptoms.

The Modified Duke's criteria are necessary to diagnose infective endocarditis. These include major and minor criteria. Diagnosis of IE is made by the prescence of either 2 major criteria or one major and three minor or five minor criteria. These criteria have a lower sensitivity in prosthetic valve endocarditis, pacemaker and defibrillator lead IE. In these scenarios an echocardiogram is usually normal or inconclusive in up to $30 \%$ of cases. [2] The modified Duke's criteria are as follows: [2] 
Table 1. Major and Minor Duke's Criteria.

\begin{abstract}
Major Duke's Criteria
Positive blood culture for typical Infective Endocarditis organisms (Streptococcus viridans or bovis, HACEK, Staphylococcus aureus without other primary site, enterococcus), from two separate blood cultures or two positive cultures from samples drawn $>$ twelve hours apart, or three or a majority of four separate cultures of blood (first and last sample drawn 1 hour apart)

Echocardiogram with oscillating intracardiac mass on valve or supporting structures, in the path of regurgitant jets, or on implanted material in the absence of an alternative anatomic explanation, or abscess, or new partial dehiscence of prosthetic valve or new valvular regurgitation
\end{abstract}

Minor Duke's Criteria

Predisposing heart condition or intravenous drug use

Temperature $>38.0^{\circ} \mathrm{C}\left(100.4^{\circ} \mathrm{F}\right)$

Vascular phenomena: arterial emboli, pulmonary infarcts, mycotic aneurysms, intracranial bleed, conjunctival hemorrhages, Janeway lesions Immunologic phenomena: glomerulonephritis, Osler nodes, Roth spots, rheumatoid factor

Microbiological evidence: positive blood culture but does not meet a major criterion as noted above or serological evidence of active infection with organism consistent with endocarditis (excluding coag neg staph, and other common contaminants)

Echocardiographic findings: consistent with endocarditis but do not meet a major criterion as noted above.

Risk factors for infective endocarditis could be cardiac or non cardiac. Non cardiac risk factors include IVDA, immunosuppression, recent surgical or dental procedures and indwelling intravenous catheters [2]. Cardiac risk factors includecardiac devices, valvular heart disease, congenital heart diseases, previous endocarditis and rheumatic heart disease. The latter is the commonest cardiac risk factor in developing countries whilstmitral valve prolapse is the most commonone in developed countries. [2] The major risk factor in our study was prosthetic valves at $19.3 \%$.

The most common organism leading to infective endocarditis is Staphylococcus aureus. Other organisms include viridians strep, Streptococcus gallolyticus, HACEK organisms and community acquired enterococci. Culture negative endocarditis is endocarditis with no definite aietiology following inoculation of at least three independently obtained blood cultures with negative cultures after five days. Negative blood cultures and persistent fever with one or more clinical findings consistent with infective endocarditis - stroke or other emboli or vegetation on echo and no clear microbiological diagnosis is diagnosed as culture negative endocarditis. [2] Culture negative endocarditis was seen in $12.3 \%$ (7) of our patients.

Infective endocarditis can lead to several organ complications. Cardiac complications include heart failure, valvular insufficiency, perivalvular abscess, pericarditis and intracardiac fistula. Neurological complications include stroke; haemorrhagic or embolic, brain abscess and meningitis. Renal complications include abscesses, glomerulonephriris and drug induced interstitial nephritis. Musculoskeletal complications include vertebral osteomyelitis and septic arthritis. Embolic complications are seen in $25 \%$ patients at the time of diagnosis. [2]

Acute native valve endocarditis is aggressive and frequently involves normal valves with no structural defects. It is most commonly due to Staphyloccoccal aureus or Group
B streptococci. Subacute native valve endocarditis affects abnormal valves and carries a more indolent course. Alpha haemolytic streptococci and enterococci are the commonest causative organisms. [2]

Prosthetic valve endocarditis accounts for $10-20 \%$ of infective endocarditis. $5 \%$ of mechanical and bioprosthetic valves become infected. Mechanical valves are usually infected within the first three months of implantation and bioprosthetic valves usually become infected within the first year. Mitral valves are more susceptible. [2] Our rate of prosthetic valve infections was $19.3 \%$ (11) with multiple different organisms being cultured as follows: Staphylococcus aureus, enterococci, viridans streptococci, other gram positive organsims andgram negative organisms. Pacemakers and implantable cardioverter defibrillators (ICDs) are usually infected within months of implantation. Staphylococci; both coagulase positive and negative are responsible for $75 \%$ of pacemaker infections. The generator pocket is the most common site for infection. The proximal leads or the portion of the lead in direct contact with endocardium can also become infected. The latter is the most serious type of infection. [2] In our cohort, previous cardiac interventions were responsible for $5.3 \%$ of infective endocarditis with Streptococcus sanguinis, enterococci, and Staphlococcus epidermidis being the most common organisms.

Intravenous drug related infective endocarditis is more common in males with Staphylococcus aureus being the commonest organism in more than half of the cases. This is followed by streptococci, enterococci, fungi and gram negative organsisms. The majority of right sided infective endocarditis occur in intravenous drug users; the tricuspic valve being more often affected than the pulmonary valve. In isolated tricuspid endocarditis symptoms are usually acute, there are no heart murmurs or peripheral stigmata of infective endocarditis and blood cultures are usually positive. [2] In our cohort $10 \%$ of IE were IVDA related endocarditis with 
Staphylococcus aureus followed by enterococci being the causative organisms.

\section{Conclusion}

This study sheds light on our local population. Despite being a small country we had a significant number of IE cases over a seven year period. The symptoms, risk factors and causative organisms conform to internationally published data. The most common organism causing IE was Stapyhlococcus aureus as is seen in foreign countries. We had a significant rate of complications in our local population. It is important to identify the patients who would benefit from surgery as early as possible during their stay as early surgery may improve patients' outcomes. [8]

\section{References}

[1] Sexton D, Fowler V. Clinical manifestations and evaluation of adults with suspected native valve endocarditis [Internet]. http://www.uptodate.com. 2016 [cited 15 August 2016]. Available from: http://Clinical manifestations and evaluation of adults with suspected native valve endocarditis

[2] Brusch J. Infective Endocarditis: Practice Essentials,
Background, Pathophysiology [Internet]. Emedicine.medscape.com. 2015 [cited 15 August 2016]. Available from:

http://emedicine.medscape.com/article/216650-overview.

[3] Habib G, Derumeaux G, Avierinos J, et al. Value and limitations of the duke criteria for the diagnosis of infective endocarditis. J Am Coll Cardiol. 1999;33(7):2023-2029. doi:10.1016/S0735-1097(99)00116-3.

[4] Tleyjeh IM, Steckelberg JM, Murad HS, et al. Temporal Trends in Infective Endocarditis: A Population-Based Study in Olmsted County, Minnesota. JAMA. 2005;293(24):3022-3028. doi:10.1001/jama.293.24.3022.

[5] David H. Bor, Steffie Woolhandler, Rachel Nardin, John Brusch, and David U. Himmelstein, Infective Endocarditis in the U.S., 1998-2009: A Nationwide Study June 22/29, 2005, Vol 293, No. 24.

[6] Prendergast B Tornos P. Surgery for Infective Endocarditis: Who and When? Circulation. 2010;121(9):1141-1152.

[7] Keys $T$ Infective endocarditis: Prevention, diagnosis, treatment, referral. Cleveland Clinic Journal of Medicine. 2000; 67(5):353-360.

[8] Murdoch D et al. Clinical Presentation, Etiology, and Outcome of Infective Endocarditis in the 21st Century. Archives of Internal Medicine. 2009;169(5):463. 